\title{
Fratura Le Fort I em paciente vítima de acidente motociclístico: relato de caso
}

\author{
Le Fort I fracture in a patient victim of a motorcycle accident: case report \\ Fractura Le Fort I em paciente víctima de accidente de motocicleta: relato de caso
}

Recebido: 21/09/2021 | Revisado: 30/09/2021 | Aceito: 06/10/2021 | Publicado: 09/10/2021

\author{
Vinícius Fernandes Cavalcante \\ ORCID: https://orcid.org/0000-0001-9606-2801 \\ Universidade de Fortaleza, Brasil \\ E-mail: Viniciusfernandescavalcante@gmail.com \\ Diego Learth Lima \\ ORCID: https://orcid.org/0000-0002-7158-6964 \\ Universidade de Fortaleza, Brasil \\ E-mail: Diegolearth95@gmail.com \\ Jair Queiroz de Oliveira Neto \\ ORCID: https://orcid.org/0000-0002-8857-0395 \\ Hospital Batista Memorial, Brasil \\ E-mail: jairqueiroz-neto@hotmail.com \\ Helder Cavalcante Carneiro Junior \\ ORCID: https://orcid.org/0000-0002-6800-5188 \\ Hospital Batista Memorial, Brasil \\ E-mail: helder_carneiro@ hotmail.com \\ João Eudes Teixeira Pinho Filho \\ ORCID: https://orcid.org/0000-0002-5785-5910 \\ Hospital Batista Memorial, Brasil \\ E-mail: joaoeudestexeira@hotmail.com \\ Murilo Alves Teixeira Neto \\ ORCID: https://orcid.org/0000-0003-1009-7347 \\ Hospital Batista Memorial, Brasil \\ E-mail: muriloteixeiraa@hotmail.com
}

\begin{abstract}
Resumo
Relato de caso: Paciente F.J.L., gênero masculino, 51 anos de idade, normossistêmico, compareceu ao serviço de Cirurgia e Traumatologia Buco-Maxilo-Facial do Hospital Batista Memorial, Fortaleza / CE. Durante a avaliação clínica verificou-se mobilidade de maxila, presença de degrau em pilar zigomático bilateralmente, degrau em margem infra-orbitária e diplopia monocular em olho esquerdo. Após a análise do exame imaginológico foi constatado fratura do tipo Le Fort I e do complexo zigomático e em seguida o paciente foi submetido a cirurgia sob efeito de anestesia geral para realização de osteossíntese das fraturas faciais, utilizando placas e parafusos do sistema $2.0 \mathrm{~mm}$ e $1.5 \mathrm{~mm}$. Atualmente, o paciente se encontra em acompanhamento pós-operatório de 6 meses sem queixas estéticos funcionais. Conclusão: A realização do tratamento realizado precocemente aliado ao correto planejamento das fraturas maxilofaciais minimizam significativamente os riscos de comprometimento estético-funcional, assim favorecendo o retorno precoce dos pacientes ao convívio social e minimizando os riscos de complicações pós-operatórias.
\end{abstract}

Palavras-chave: Fraturas Maxilares; Traumatismo Facial; Maxila.

\begin{abstract}
Case report: Patient F.J.L., male, 51 years old, normosystemic, attended the Maxillofacial Surgery and Traumatology Service of Hospital Batista Memorial, Fortaleza / CE. During the clinical evaluation, mobility of the maxilla, the presence of a step in the zygomatic pillar bilaterally, a step in the infraorbital margin and monocular diplopia in the left eye were verified. After the analysis of the imaging exam, a Le Fort I and zygomatic complex fracture was found, and then the patient underwent surgery under general anesthesia to perform osteosynthesis of the facial fractures, using plates and screws of the $2.0 \mathrm{~mm}$ and 1.5 system $\mathrm{mm}$. Currently, the patient is in postoperative follow-up for 6 months without functional aesthetic complaints. Conclusion: Early treatment combined with correct planning of maxillofacial fractures significantly minimizes the risks of esthetic-functional impairment, thus favoring the early return of patients to social life and minimizing the risk of postoperative complications.
\end{abstract}

Keywords: Jaw fractures; Facial trauma; Jaw.

\section{Resumen}

Caso clínico: Paciente F.J.L., varón, 51 años, normosistémico, acudió al Servicio de Cirugía Maxilofacial y Traumatología del Hospital Batista Memorial, Fortaleza / CE. Durante la evaluación clínica se verificó la movilidad del maxilar, la presencia de un escalón en el pilar cigomático bilateralmente, un escalón en el margen infraorbitario y 
diplopía monocular en el ojo izquierdo. Tras el análisis del examen de imagen, se encontró la fractura de Le Fort I y del complejo cigomático, y luego se intervino al paciente bajo anestesia general para realizar la osteosíntesis de las fracturas faciales, utilizando placas y tornillos del sistema de $2,0 \mathrm{~mm}$ y $1,5 \mathrm{~mm}$. Actualmente, el paciente se encuentra en seguimiento postoperatorio durante 6 meses sin quejas estéticas funcionales. Conclusión: El tratamiento precoz combinado con una correcta planificación de las fracturas maxilofaciales minimiza significativamente los riesgos de deterioro estético-funcional, favoreciendo así el retorno temprano de los pacientes con vida social y mínimo riesgo de complicaciones postoperatorias.

Palabras clave: Fracturas Maxilares; Trauma facial; Mandíbula.

\section{Introdução}

O trauma facial é uma das agressões mais complexas encontradas em centros de trauma devido às consequências emocionais e à possibilidade de deformidade, dentre as etiologias do trauma facial os acidentes automotores se consideram uma das causas mais significativas (Sastry et al.,1995; Erdmann et al.,2008). Necessitando de uma criteriosa avaliação e condução de rápido início para minimizar possíveis complicações como limitações definitivas ou óbito (ACS, 1997).

As fraturas do terço médio da face envolvem relevantes estruturas ósseas da face como a maxila, zigoma e o complexo naso órbito etimoidal (NOE), são classificadas em: fraturas Le fort I, II ou III, fraturas do complexo zigomático maxilar, fraturas de arco zigomático ou fraturas do tipo NOE (Matthew, Agrawal, Truonh, 2017).

A classificação de Le Fort para fraturas faciais foi relatada em 1901 pelo cirurgião francês Rene Le Fort, e é aplicada até os dias atuais para a descrição de fraturas do terço médio da face, sendo denominadas de fraturas Le Fort I, II e III (Bagheri et al.,2005).

Monnazzi et al., $2002 \mathrm{em}$ seu estudo demonstrou que normalmente as fraturas do tipo Le Fort ocorrem em um maior número de formas associadas entre si ou associadas com outros tipos de faturas em face, devido a grandes sinergias dos resultados em traumas maxilofaciais. Em que na maioria dos casos acomete o complexo zigomático, seio frontal, mandíbula, nariz, complexo orbitário, naso-órbito-etmoidal (NOE) e arco zigomático, podendo estar ou não associadas umas às outras.

Segundo a analise de 194 casos no estudo de Silva et al., 2011 o principal fator etiológico dos traumas faciais está relacionado a acidentes de trânsito, com destaque para os acidentes motociclísticos, seguido da violência interpessoal com predomínio do gênero masculino e pico de incidência entre 21 e 30 anos. Vale salientar, que as etiologias supracitadas sofrem influência de fatores sociais, econômicos e culturais; além de variações de gênero, faixa etária e etiologia a depender do local do estudo (Stolz et al.,2011).

As fraturas do tipo Le Fort I, também conhecida como fratura de Guerin ou transversa, resultam em mobilidade da maxila, podendo ou não estar associada a má oclusão dentária. Em relação ao mecanismo do trauma, geralmente estão associadas a trauma de baixa sinergia e consequentemente com menor gravidade quando comparado as fraturas Le Fort II e III (Bradley, Lauren, 2017).

\section{Metodologia}

O intuito deste trabalho é relatar e detalhar o tratamento cirúrgico de um paciente vítima de trauma bucomaxilofacial com fraturas em terço médio de face de forma descritiva e qualitativa (Pereira et al.,2018). Por meio de uma breve revisão da literatura, desde aspectos epidemiológicos do trauma a métodos de diagnóstico e tratamento.

\section{Relato de Caso}

Paciente F.J.L., gênero masculino, 51 anos de idade, normossistêmico, compareceu ao serviço de Cirurgia e Traumatologia Buco-Maxilo-Facial do Hospital Batista Memorial - Fortaleza / CE, Brasil, após acidente automobilístico com 
trauma facial. Ao exame clínico verificou-se: mobilidade de maxila, degrau em pilar zigomático bilateralmente, degrau em margem infra-orbitária e diplopia monocular em olho esquerdo.

$\mathrm{Na}$ avaliação radiográfica, foi evidenciado uma fratura Le Fort I associado a fratura do complexo zigomático maxilar e arco zigomático, com diástase em sutura fronto-zigomática e deslocamento da sutura esfeno-zigomática esquerda. As imagens das tomografias computadorizadas (TC) e do estado clinico inicial estão demonstradas nas Figura 1.

Figura 1 - A: Imagem pré-operatória do paciente; B: TC corte coronal demonstrando as fraturas em sutura fronto-zigomática, em assoalho infraorbitária e pilares zigomáticos; C: TC corte axial, demonstrando o deslocamento da sutura esfenozigomatica. D: Projeção 3D tomográfica, demonstrando as fraturas em sutura fronto-zigomática, assoalho de orbita, pilares zigomáticos e maxila.

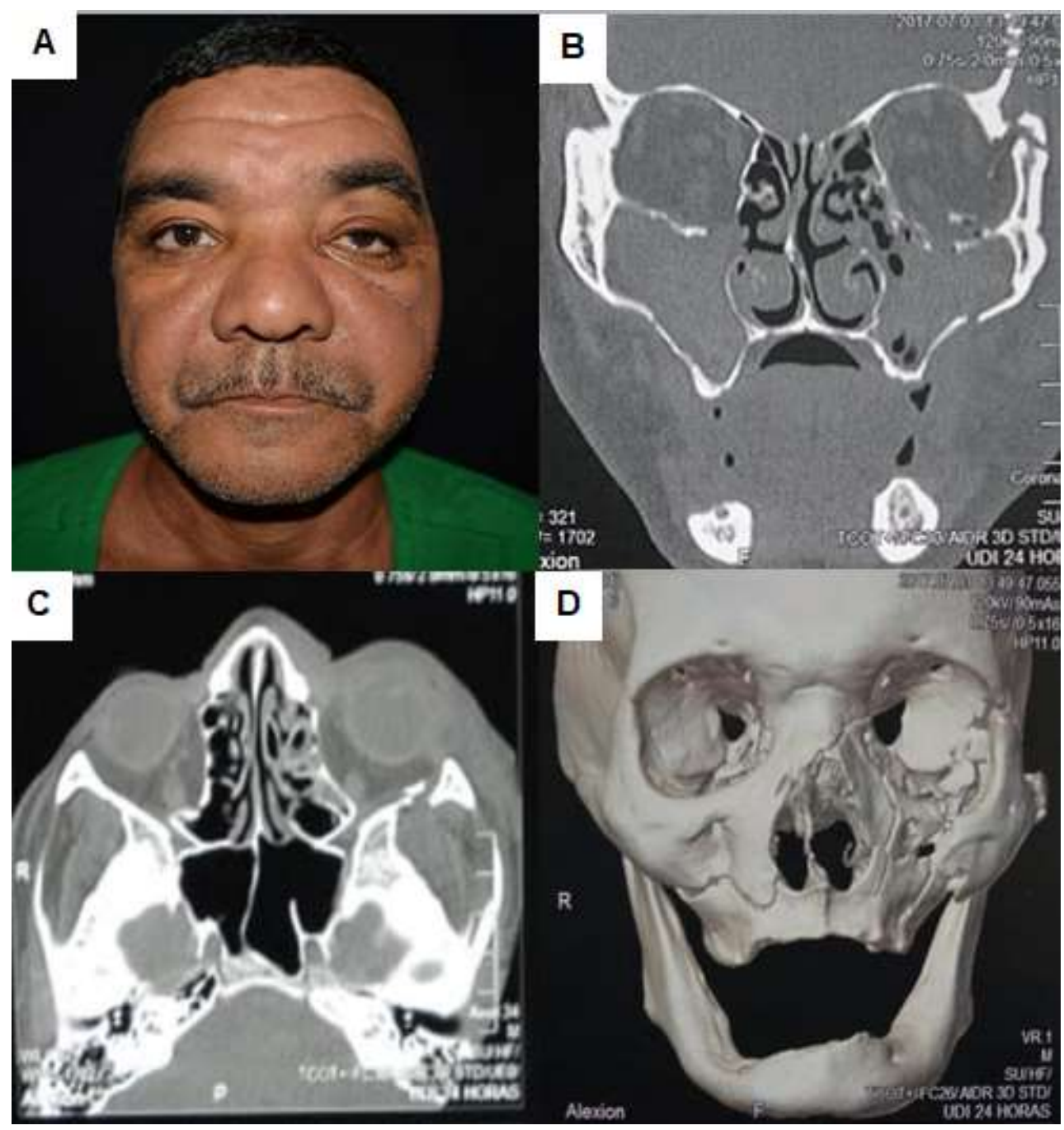

Fonte: serviço de CTBMF do Hospital Batista Memorial.

Após a avaliação clínica e radiográfica, o paciente foi submetido a cirurgia, sob anestesia geral, para osteossíntese das fraturas por meio de uma redução anatômica e fixação interna rígida utilizando placas e parafusos no sistema $1.5 \mathrm{~mm}$ e $2.0 \mathrm{~mm}$.

Para a fixação das fraturas foi realizado os seguintes acessos para gerar as reduções e osteossínteses. $\mathrm{O}$ acesso superciliar para a sutura fronto-zigomática com placas e parafusos do sistema 1.5mm (Figura 2-A); um acesso subtarsal, em região do ferimento corto-contuso apresentado, em margem infra-orbitária com placas e parafusos do sistema 1.5mm (Figura 
2-B) e os acessos intra-orais bilaterais em fundo vestíbulo da maxila para redução da fratura Le Fort I com placas e parafusos do sistema 2.0mm (Figura 2- C e D).

Figura 2 Imagens durante o transoperatório após a fixação. A: acesso superciliar frontozigomático B: acesso subtarsal. C: acesso intra-oral em fundo de vestíbulo do lado esquerdo. D: acesso intra-oral em fundo de vestíbulo do lado direito.

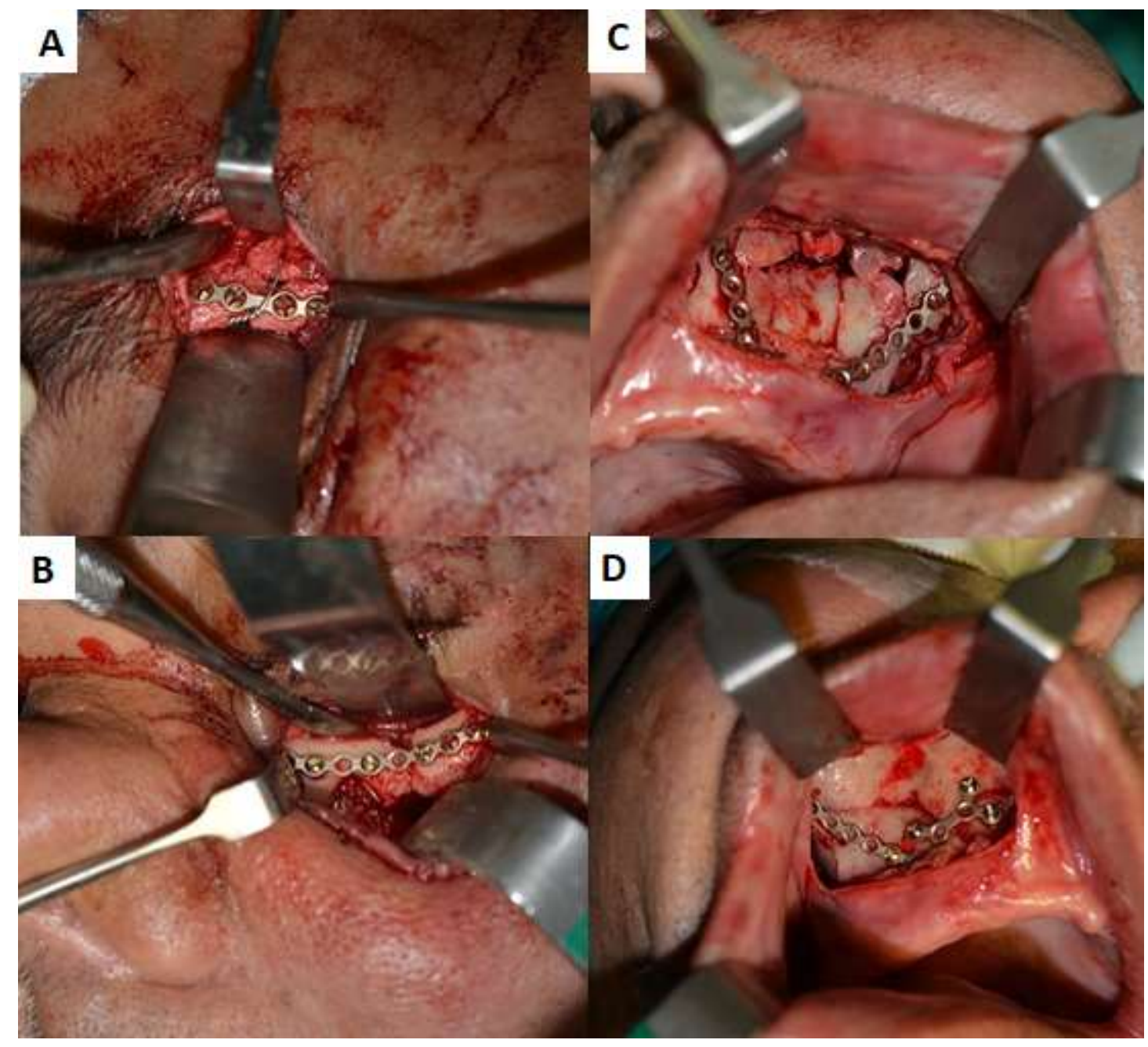

Fonte: serviço de CTBMF do Hospital Batista Memorial.

As suturas foram realizadas por planos, inicialmente com vicryl nos acessos supraorbital, subtarsal e vestibular da maxila, e finalizado com nylon em região superficial de pele e em mucosa jugal. Atualmente o paciente encontra-se em acompanhamento pós-operatório de 6 meses sem queixas estéticas ou funcionais e restabelecimento da função ocular, conforme a Figura 3. 
Figura 3 Imagens do pós-operatório de 6 meses. A: Vista frontal. B: Projeção 3D frontal tomografia pós-operatória.

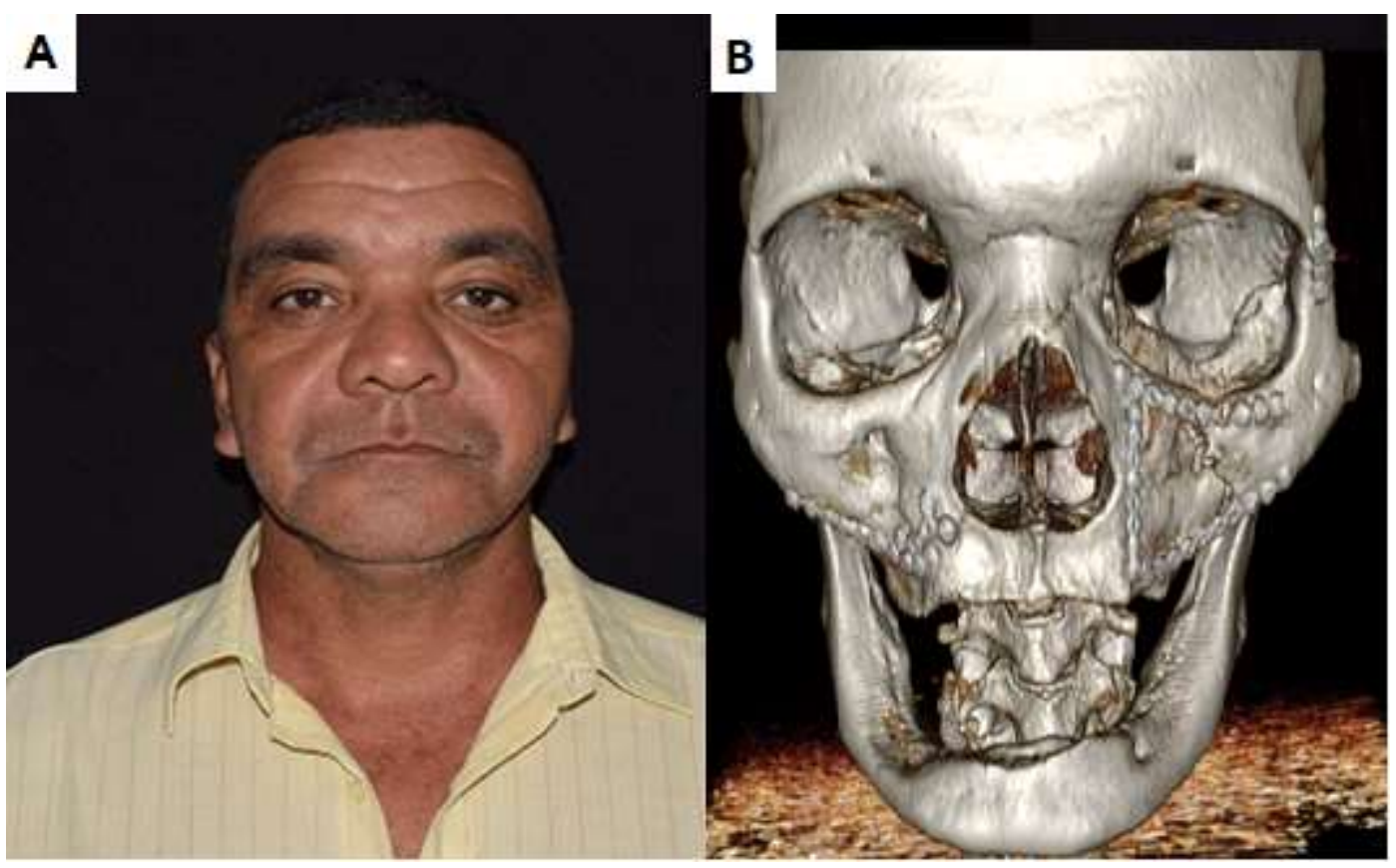

Fonte: serviço de CTBMF do Hospital Batista Memorial.

\section{Discussão}

O propósito do tratamento das fraturas faciais de maneira geral é restaurar a arquitetura do esqueleto facial. Um erro no correto posicionamento do osso fraturado no decorrer de uma redução, podem resultar em sequelas que são de difícil correção em segundo momento cirúrgico (Jardim et al., 2013).

No estudo de Monnazzi et al. 2002, que avaliou complicações tardias em fraturas do tipo Le Fort, 58\% do total de casos do estudo ocorreu concomitante a outras fraturas faciais. Apresentando um predomínio para as fraturas do complexo zigomático (29\%) seguidos pelas fraturas do seio frontal (22\%). Predomínio que se assemelha com o caso descrito no presente trabalho.

O estudo de Carinci et al. 2008 corrobora com o presente caso em relação a associação das demais fraturas faciais com a fraturas Le Fort; incluindo as fraturas naso-orbito-etmóides (NOE), zigomático-maxilares e dento-alveolares como as mais prevalentes.

A fratura do osso zigomático encontra-se frequentemente relacionado as fraturas Le Fort e dentre os motivos para tal associação, podemos citar as suas projeções anatómicas robustas, que contribui para o sistema de contraforte vertical da face média, se articulando com os ossos faciais circundantes de maior fragilidade, podendo resultar no envolvimento das suturas frontal zigomática (ZF), a zigomático-temporal (ZT) e a zigomático-maxilar (ZM) (Fraioli, 2008)

Ainda em relação as lesões concomitantes, os pacientes com fraturas faciais devem ser avaliados quanto a possíveis lesões cranianas e em coluna cervical, principalmente nos impactos de alta sinergia. No caso das Fraturas Le Fort, estes riscos estão comumente mais associados aos tipos II e III em relação ao tipo I (Bradley, Lauren, 2017), como no caso descrito, em que não ocorreu tais comorbidades.

O diagnóstico das fraturas Le Fort é realizado através de exames físicos e imaginológicos, no qual são fundamentais para reconhecer e realizar o correto diagnóstico desses tipos de fraturas, e assim permitir o tratamento apropriado. Clinicamente as fraturas Le Fort I demonstram alguns sinais e sintomas como mobilidade, principalmente ao manipular a maxila, além de dor, edema em nível do lábio superior, equimoses em fundo de vestíbulo anterior da maxila e em região 
palatina posterior, enfisema nos tecidos moles das regiões genianas e zigomáticas (devido ao envolvimento das paredes dos seios maxilares), além de uma alteração na oclusão dentária com uma possível mordida aberta anterior ao se tratar de indivíduos dentados (Mardones et al., 2011).

Para cada tipo de fratura em face média há considerações únicas durante o diagnóstico e tratamento. Porém de maneira geral a base do tratamento em fraturas associadas a um deslocamento ósseo, consiste na redução aberta e fixação interna com placas e parafusos do sistema $1.5 \mathrm{~mm}$ e $2.0 \mathrm{~mm}$ (McRae \& Frodel, 2000).

Mesmo em pacientes edêntulos, o reestabelecimento das relações maxilo-mandibulares durante a redução aberta é essencial em fraturas de Le Fort (Oliveira-Campos et al.,2016). Esse restabelecimento maxilo-mandibular é realizado através da inserção e fixação da própria prótese do paciente, e em casos de ausência protética pode-se utilizar a técnica de goteira acrílica ou Goteira de Gunning (Crawley et al., 1997). E na impossibilidade de restabelecer da maneira supracitada, como no caso do presente relato clínico, a redução anatômica surge como alternativa para restabelecimento do correto posicionamento das estruturas envolvidas (Oliveira-Campos et al.,2016).

Em fraturas Le Fort I, a maloclusão é uma das principais complicações que podem acometer o paciente (Buehler, Tannyhill., 2003). Nessas fraturas, as abordagens cirúrgicas intra-orais são selecionadas para restabelecimento do posicionamento adequado dos segmentos fraturados com mínimo comprometimento estético e baixo índice de complicações (Oliveira-Campos et al.,2016), entretanto quando lesões concomitantes são encontradas, faz-se necessário na maioria dos casos a associação com acessos transcutâneos, podendo ser o acesso subtarsal, subciliar e transconjutival. Em casos de lacerações presentes, pode-se utilizar do ferimento prévio para acesso a devida região para redução e fixação (Colombo et al.,2017), como descrito ao caso em questão.

No estudo de Simon et al, onde foi proposto a redução e fixação das fraturas em pilar canino e pilar zigomático com placas e parafusos do sistema 2.0, em um tratamento cirúrgico de fraturas Le Fort I e Le Fort II, demostrou sucesso na obtenção de um bom resultado estético e funcional ao paciente, favorecendo o retorno da oclusão aos padrões de normalidade.

\section{Conclusão}

As fraturas Le Fort são fraturas faciais específicas ocasionadas após um trauma do tipo contuso. Apesar de apresentarem baixas taxas de mortalidade, essas lesões estão frequentemente associadas a outras lesões graves na cabeça e pescoço. Dessa maneira é de suma importância o diagnóstico das fraturas faciais com rapidez e precisão para obter um plano de tratamento ideal, minimizando possíveis complicações no pós operatório.

O presente trabalho aponta a relevância da necessidade do diagnóstico correto e da abordagem precoce na área de Traumatologia Buco-Maxilo-Facial, a fim de evitar maiores complicações. Por mais que constitua um tema bem estabelecido nas bases bibliográficas, é de grande importância a realização de estudos futuros que possam aperfeiçoar a conduta de casos semelhantes.

\section{Referências}

ACS. (1997). Advanced Trauma Life Support for Doctors. American Colege of Surgeons (ACS).

Bagheri, S. C., Holmgren, E., Kademani, D., Hommer, L., Bell, R. B. Potter, B. et al. (2005). Comparison of the severity of bilateral Le Fort injuries in isolated midface trauma. Journal of oral and maxillofacial surgery. 63(8):1123-9.

Bradley, J. P., \& Lauren, M. T. (2017). Le Fort fractures: a collective review. Bulletin of Emergency \& Trauma. 5(4): 221-30.

Buehler J. A., \& Tannyhill, R. J. (2003). Complications in the treatment of midfacial fractures. Oral Maxillofacial Surgery Clinics North Am.15(2):195-212.

Carinci F., Zollino I., Arduin L., Brunelli G., Pagliaro F., \& Cenzi R. (2008). Midfacial fractures: A scoring method and validation on 117 patients. Eur J Trauma Emerg Surg. 34(4):385-90. 
Research, Society and Development, v. 10, n. 13, e178101320844, 2021

(CC BY 4.0) | ISSN 2525-3409 | DOI: http://dx.doi.org/10.33448/rsd-v10i13.20844

Colombo L. T, Santos G. M, Gonçalves P. Z, Fabris A. L. S., Souza F. Á. \& Faverani L. P. (2017). Fratura do complexo zigomaticomaxilar por agressão física: relato de caso. Archives Of Health Investigation. 6(8):390-393.

Crawley W. A., Azman P., Clark N., Robertson B., Slezak S. Vander C. K., et al. (1997). The edentulous Le Fort fracture. J CraniofacSurg. 8(4):298-307.

Erdmann D., Follmar K. E., Debruijn M., et al. (2008). A retrospective analysis of facial fracture etiologies. Ann Plast Surg. 60(4):398-403.

Fraioli R. E., Branstetter B. F., \& Deleyiannis F. W. (2008). Facial fractures: Beyond le fort. Otolaryngol Clin North Am. 41(1):51-76.

Jardim E. C. G., Júnior S., Ferreira J., Melo R. L. D., Mendonça J. C. G. D. Faverani L. P., et al. (2013). Combinação de técnicas para tratamento cirúrgico de fratura do complexo zigomático-maxilar: relato de caso. Archives of Health Investigation. 2(3): 33-36.

Mardones M. M., Fernández T. M. D. L. Á., Bravo A. R., Pedemonte T. C. \& Ulloa M. C. (2011). Traumatología maxilo facial: Diagnostico y tratamiento. Revista Medica Clinica Las Condes. 22(5): 607-616.

Matthew L., Agrawal N., \& Truonh T. A. (2017). Midface Fractures II. Semin Plast Surg 31(2):94- 99.

McRae M. \& Frodel, J. (2000). Midface fractures. Facial Plast Surg. 16(2):107-13.

Monnazzi M. S., Hochuli-Vieira E., Gabrielli M. A. C., Gabrielli M. F. R. \& Pereira Filho V. A. (2002). Avaliação de complicações tardias em fraturas maxilares do tipo Le Fort. Rev Fac Odontol Bauru. 10(4):257-262.

Oliveira-Campos G. H., Lauriti L., Yamamoto M. K., Junior R. C. \& Luz J. G. (2016). Trends in le fort fractures at a South American trauma care center: Characteristics and management. J Maxillofac Oral Surg. 15(1):32-37.

Pereira A. S. et al. (2018). Metodologia da pesquisa científica. UFSM.

Sastry S. M., Sastry C. M., Paul B. K. \& Bain L. (1995). Champion H. R. Leading causes of facial trauma in the major trauma outcome study. Plast Reconstr Surg. 95:196-7.

Silva J. J. D. L., Lima A. A. A. S., Melo I. F. S., Maia R. C. L. \& Pinheiro Filho T. R. D. C. (2011). Trauma facial: análise de 194 casos. Rev. bras. cir. Plást. 26(1):37-41.

Simon, M. E. S., Momesso, G. A. C., Silva, W. P. P., Delanora, L. A., Delanora, L. A., Santos A. M. S., Lima N. T. J, Faverani, L. P. \& Paiva, L. C. A. (2020). Tratamento cirúrgico de fraturas Le Fort I e Le Fort II em vítima de trauma por acidente motociclístico: relato de caso. Archives of Helth Investigation. 9(6), 546-549.

Stolz A. D. S. B., Meller F. B., Quesada G. A., Bergoli C., Escobar C. A. B., \& Martins E. M. (2011). Análise epidemiológica de fraturas bucomaxilofaciais em pacientes atendidos no Hospital Universitário de Santa Maria-HUSM: um estudo retrospectivo. Revista Odontológica do Brasil Central. 20(53):129-135. 